\title{
INEQUALITIES FOR ORTHORNORMAL FAMILIES OF VECTORS IN INNER PRODUCT SPACES RELATED TO BUZANO'S, RICHARD'S AND KUREPA'S RESULTS
}

\author{
S. S. DRAGOMIR
}

Abstract. Some inequalities for families of orthornormal vectors in inner product spaces that are related with Buzano's, Richard's and Kurepa's results are given.

\section{Introduction}

In [3], M. L. Buzano obtained the following extension of the celebrated Schwarz's inequality in a real or complex inner product space $(H ;\langle\cdot, \cdot\rangle)$ :

$$
|\langle a, x\rangle\langle x, b\rangle| \leq \frac{1}{2}[\|a\|\|b\|+|\langle a, b\rangle|]\|x\|^{2},
$$

for any $a, b, x \in H$.

It is clear that the above inequality becomes, for $a=b$, the Schwarz's inequality

$$
|\langle a, x\rangle|^{2} \leq\|a\|^{2}\|x\|^{2}, \quad a, x \in H ;
$$

in which the equality holds if and only if there exists a scalar $\lambda \in \mathbb{K}(\mathbb{R}, \mathbb{C})$ so that $x=\lambda a$.

As noted by M. Fujii and I. Kubo in [5], where they provided a simple proof of (1.1) on using orthogonal projection arguments, the case of equality holds in (1.1) if

$$
x=\left\{\begin{array}{l}
\alpha\left(\frac{a}{\|a\|}+\frac{\langle a, b\rangle}{\langle a a, b\rangle \mid} \cdot \frac{b}{\|b\|}\right), \text { when }\langle a, b\rangle \neq 0 \\
\alpha\left(\frac{a}{\|a\|}+\beta \cdot \frac{b}{\|b\|}\right), \quad \text { when }\langle a, b\rangle=0,
\end{array}\right.
$$

where $\alpha, \beta \in \mathbb{K}$.

As noted by T. Precupanu in [8], independently of Buzano, U. Richard [9] obtained the following similar inequality holding in real inner product spaces:

$$
\frac{1}{2}\|x\|^{2}[\langle a, b\rangle-\|a\|\|b\|] \leq\langle a, x\rangle\langle x, b\rangle \leq \frac{1}{2}\|x\|^{2}[\langle a, b\rangle+\|a\|\|b\|] .
$$

Received February 15, 2005.

2000 Mathematics Subject Classification. 46C05, 46C09, 26D15.

Key words and phrases. Schwarz's inequality, Buzano's inequality, Richard's inequality, Kurepa's inequality, inner products, integral inequalities. 
In [7], J. Pečarić gave a simple direct proof of (1.4) without mentioning the work of either Buzano of Richard, but tracked down the result, in a particular form, to an earlier paper of C. Blatter [1].

In [4], the author has improved Buzano's inequality by showing that, in fact, one has:

$$
\begin{aligned}
|\langle a, x\rangle\langle x, b\rangle| & \leq\left|\langle a, x\rangle\langle x, b\rangle-\frac{1}{2}\langle a, b\rangle\|x\|^{2}\right|+\frac{1}{2}|\langle a, b\rangle|\|x\|^{2} \\
& \leq \frac{1}{2}[\|a\|\|b\|+|\langle a, b\rangle|]\|x\|^{2} .
\end{aligned}
$$

In the same paper, the author has also improved the celebrated Kurepa inequality for the complexification of a real inner product space, namely, the inequality [6]

$$
\left|\langle z, x\rangle_{\mathbb{C}}\right|^{2} \leq \frac{1}{2}\|x\|^{2}\left[\|z\|_{\mathbb{C}}^{2}+\left|\langle z, \bar{z}\rangle_{\mathbb{C}}\right|\right] \leq\|x\|^{2}\|z\|_{\mathbb{C}}^{2}
$$

where $x \in H,(H ;\langle\cdot, \cdot\rangle)$ is a real space, $z \in H_{\mathbb{C}},\left(H_{\mathbb{C}},\langle\cdot, \cdot\rangle_{\mathbb{C}}\right)$ is the complexification of $(H ;\langle\cdot, \cdot\rangle)$, and $\bar{z}$ is the conjugate vector of $z$.

The refinement of Kurepa's result (1.6) obtained in [4] is as follows:

$$
\begin{aligned}
\left|\langle z, x\rangle_{\mathbb{C}}\right|^{2} & \leq\left|\langle z, x\rangle_{\mathbb{C}}^{2}-\frac{1}{2}\langle z, \bar{z}\rangle_{\mathbb{C}}\|x\|^{2}\right|+\frac{1}{2}\left|\langle z, \bar{z}\rangle_{\mathbb{C}}\right|\|x\|^{2} \\
& \leq \frac{1}{2}\|x\|^{2}\left[\|z\|_{\mathbb{C}}^{2}+\left|\langle z, \bar{z}\rangle_{\mathbb{C}}\right|\right] \\
& \leq\|x\|^{2}\|z\|_{\mathbb{C}}^{2}
\end{aligned}
$$

with the same assumptions as above.

The main aim of the present paper is to obtain similar results for families of orthonormal vectors in $(H ;\langle\cdot, \cdot\rangle)$, real or complex space, that are naturally connected with the celebrated Bessel inequality.

\section{A Generalisation for Orthonormal Families}

We say that the finite family $\left\{e_{i}\right\}_{i \in I}$ ( $I$ is finite) of vectors is orthonormal if $\left\langle e_{i}, e_{j}\right\rangle=$ 0 if $i, j \in I$ with $i \neq j$ and $\left\|e_{i}\right\|=1$ for each $i \in I$. The following result may be stated:

Theorem 1. Let $(H ;\langle\cdot, \cdot\rangle)$ be an inner product space over the real or complex number field $\mathbb{K}$ and $\left\{e_{i}\right\}_{i \in I}$ a finite orthonormal family in $H$. Then for any $a, b \in H$, one has the inequality:

$$
\left|\sum_{i \in I}\left\langle a, e_{i}\right\rangle\left\langle e_{i}, b\right\rangle-\frac{1}{2}\langle a, b\rangle\right| \leq \frac{1}{2}\|a\|\|b\| .
$$

The case of equality holds in (2.1) if and only if

$$
\sum_{i \in I}\left\langle a, e_{i}\right\rangle e_{i}=\frac{1}{2} a+\left(\sum_{i \in I}\left\langle a, e_{i}\right\rangle\left\langle e_{i}, b\right\rangle-\frac{1}{2}\langle a, b\rangle\right) \cdot \frac{b}{\|b\|^{2}} .
$$


Proof. It is well known that, for $e \neq 0$ and $f \in H$, the following identity holds:

$$
\frac{\|f\|^{2}\|e\|^{2}-|\langle f, e\rangle|^{2}}{\|e\|^{2}}=\left\|f-\frac{\langle f, e\rangle e}{\|e\|^{2}}\right\|^{2} .
$$

Therefore, in Schwarz's inequality

$$
|\langle f, e\rangle|^{2} \leq\|f\|^{2}\|e\|^{2}, \quad f, e \in H
$$

the case of equality, for $e \neq 0$, holds if and only if

$$
f=\frac{\langle f, e\rangle e}{\|e\|^{2}}
$$

Let $f:=2 \sum_{i \in I}\left\langle a, e_{i}\right\rangle e_{i}-a$ and $e:=b$. Then, by Schwarz's inequality (2.4), we may state that

$$
\left|\left\langle 2 \sum_{i \in I}\left\langle a, e_{i}\right\rangle e_{i}-a, b\right\rangle\right|^{2} \leq\left\|2 \sum_{i \in I}\left\langle a, e_{i}\right\rangle e_{i}-a\right\|^{2}\|b\|^{2}
$$

with equality, for $b \neq 0$, if and only if

$$
2 \sum_{i \in I}\left\langle a, e_{i}\right\rangle e_{i}-a=\left\langle 2 \sum_{i \in I}\left\langle a, e_{i}\right\rangle e_{i}-a, b\right\rangle \frac{b}{\|b\|^{2}} .
$$

Since

$$
\left\langle 2 \sum_{i \in I}\left\langle a, e_{i}\right\rangle e_{i}-a, b\right\rangle=2 \sum_{i \in I}\left\langle a, e_{i}\right\rangle\left\langle e_{i}, b\right\rangle-\langle a, b\rangle
$$

and

$$
\begin{aligned}
\left\|2 \sum_{i \in I}\left\langle a, e_{i}\right\rangle e_{i}-a\right\|^{2} & =4\left\|\sum_{i \in I}\left\langle a, e_{i}\right\rangle e_{i}\right\|^{2}-4 \operatorname{Re}\left\langle\sum_{i \in I}\left\langle a, e_{i}\right\rangle e_{i}, a\right\rangle+\|a\|^{2} \\
& =4 \sum_{i \in I}\left|\left\langle a, e_{i}\right\rangle\right|^{2}-4 \sum_{i \in I}\left|\left\langle a, e_{i}\right\rangle\right|^{2}+\|a\|^{2} \\
& =\|a\|^{2},
\end{aligned}
$$

hence by (2.5) we deduce the desired inequality (2.1).

Finally, as (2.2) is equivalent to

$$
\sum_{i \in I}\left\langle a, e_{i}\right\rangle e_{i}-\frac{a}{2}=\left(\sum_{i \in I}\left\langle a, e_{i}\right\rangle\left\langle e_{i}, b\right\rangle-\frac{1}{2}\langle a, b\rangle\right) \frac{b}{\|b\|^{2}},
$$

hence the equality holds in (2.1) if and only if (2.2) is valid. 
The following result is well known in the literature as Bessel's inequality

$$
\sum_{i \in I}\left|\left\langle x, e_{i}\right\rangle\right|^{2} \leq\|x\|^{2}, \quad x \in H
$$

where, as above, $\left\{e_{i}\right\}_{i \in I}$ is a finite orthonormal family in the inner product space $(H ;\langle\cdot, \cdot\rangle)$

If one chooses $a=b=x$ in (2.1), then one gets the inequality

$$
\left.\left|\sum_{i \in I}\right|\left\langle x, e_{i}\right\rangle\right|^{2}-\frac{1}{2}\|x\|^{2} \mid \leq \frac{1}{2}\|x\|^{2},
$$

which is obviously equivalent to Bessel's inequality (2.7). Therefore, the inequality (2.1) may be regarded as a generalisation of Bessel's inequality as well.

Utilising the Bessel and Cauchy-Bunyakovsky-Schwarz inequalities, one may state that

$$
\left|\sum_{i \in I}\left\langle a, e_{i}\right\rangle\left\langle e_{i}, b\right\rangle\right| \leq\left[\sum_{i \in I}\left|\left\langle a, e_{i}\right\rangle\right|^{2} \sum_{i \in I}\left|\left\langle b, e_{i}\right\rangle\right|^{2}\right]^{\frac{1}{2}} \leq\|a\|\|b\|
$$

A different refinement of the inequality between the first and the last term in (2.8) is incorporated in the following:

Corollary 1. With the assumption of Theorem 1, we have

$$
\begin{aligned}
\left|\sum_{i \in I}\left\langle a, e_{i}\right\rangle\left\langle e_{i}, b\right\rangle\right| & \leq\left|\sum_{i \in I}\left\langle a, e_{i}\right\rangle\left\langle e_{i}, b\right\rangle-\frac{1}{2}\langle a, b\rangle\right|+\frac{1}{2}|\langle a, b\rangle| \\
& \leq \frac{1}{2}[\|a\|\|b\|+|\langle a, b\rangle|] \\
& \leq\|a\|\|b\| .
\end{aligned}
$$

Remark 1. If the space $(H ;\langle\cdot, \cdot\rangle)$ is real, then, obviously, $(2.1)$ is equivalent to:

$$
\frac{1}{2}(\langle a, b\rangle-\|a\|\|b\|) \leq \sum_{i \in I}\left\langle a, e_{i}\right\rangle\left\langle e_{i}, b\right\rangle \leq \frac{1}{2}[\|a\|\|b\|+\langle a, b\rangle] .
$$

Remark 2. It is obvious that if the family comprises of only a single element $e=\frac{x}{\|x\|}$, $x \in H, x \neq 0$, then from (2.9) we recapture the refinement of Buzano's inequality incorporated in (1.5) while from (2.10) we deduce Richard's result from (1.4).

The following corollary of Theorem 1 is of interest as well:

Corollary 2. Let $\left\{e_{i}\right\}_{i \in I}$ be a finite orthonormal family in $(H ;\langle\cdot, \cdot\rangle)$. If $x, y \in H \backslash\{0\}$ are such that there exists the constants $m_{i}, n_{i}, M_{i}, N_{i} \in \mathbb{R}, i \in I$ such that:

$$
-1 \leq m_{i} \leq \frac{\operatorname{Re}\left\langle x, e_{i}\right\rangle}{\|x\|} \cdot \frac{\operatorname{Re}\left\langle y, e_{i}\right\rangle}{\|y\|} \leq M_{i} \leq 1, \quad i \in I
$$


and

$$
-1 \leq n_{i} \leq \frac{\operatorname{Im}\left\langle x, e_{i}\right\rangle}{\|x\|} \cdot \frac{\operatorname{Im}\left\langle y, e_{i}\right\rangle}{\|y\|} \leq N_{i} \leq 1, \quad i \in I
$$

then

$$
2 \sum_{i \in I}\left(m_{i}+n_{i}\right)-1 \leq \frac{\operatorname{Re}\langle x, y\rangle}{\|x\|\|y\|} \leq 1+2 \sum_{i \in I}\left(M_{i}+N_{i}\right) .
$$

Proof. Using Theorem 1 and the fact that for any complex number $z,|z| \geq|\operatorname{Re} z|$, we have

$$
\begin{aligned}
\left|\sum_{i \in I} \operatorname{Re}\left[\left\langle x, e_{i}\right\rangle\left\langle e_{i}, y\right\rangle\right]-\frac{1}{2} \operatorname{Re}\langle x, y\rangle\right| & \leq\left|\sum_{i \in I}\left\langle x, e_{i}\right\rangle\left\langle e_{i}, y\right\rangle-\frac{1}{2}\langle x, y\rangle\right| \\
& \leq \frac{1}{2}\|x\|\|y\| .
\end{aligned}
$$

Since

$$
\operatorname{Re}\left[\left\langle x, e_{i}\right\rangle\left\langle e_{i}, y\right\rangle\right]=\operatorname{Re}\left\langle x, e_{i}\right\rangle \operatorname{Re}\left\langle y, e_{i}\right\rangle+\operatorname{Im}\left\langle x, e_{i}\right\rangle \operatorname{Im}\left\langle y, e_{i}\right\rangle,
$$

hence by (2.14) we have:

$$
\begin{aligned}
& -\frac{1}{2}\|x\|\|y\|+\frac{1}{2} \operatorname{Re}\langle x, y\rangle \\
& \leq \sum_{i \in I} \operatorname{Re}\left\langle x, e_{i}\right\rangle \operatorname{Re}\left\langle y, e_{i}\right\rangle+\sum_{i \in I} \operatorname{Im}\left\langle x, e_{i}\right\rangle \operatorname{Im}\left\langle y, e_{i}\right\rangle \\
& \leq \frac{1}{2}\|x\|\|y\|+\frac{1}{2} \operatorname{Re}\langle x, y\rangle .
\end{aligned}
$$

Utilising the assumptions (2.11) and (2.12), we have

$$
\sum_{i \in I} m_{i} \leq \sum_{i \in I} \frac{\operatorname{Re}\left\langle x, e_{i}\right\rangle \operatorname{Re}\left\langle y, e_{i}\right\rangle}{\|x\|\|y\|} \leq \sum_{i \in I} M_{i}
$$

and

$$
\sum_{i \in I} n_{i} \leq \sum_{i \in I} \frac{\operatorname{Im}\left\langle x, e_{i}\right\rangle \operatorname{Im}\left\langle y, e_{i}\right\rangle}{\|x\|\|y\|} \leq \sum_{i \in I} N_{i}
$$

Finally, on making use of $(2.15)$ - (2.17), we deduce the desired result (2.13).

Remark 3. By Schwarz's inequality, is it obvious that, in general,

$$
-1 \leq \frac{\operatorname{Re}\langle x, y\rangle}{\|x\|\|y\|} \leq 1
$$

Consequently, the left inequality in (2.13) is of interest when $\sum_{i \in I}\left(m_{i}+n_{i}\right)>0$, while the right inequality in (2.13) is of interest when $\sum_{i \in I}\left(M_{i}+N_{i}\right)<0$. 


\section{Refinements of Kurepa's Inequality}

Let $(H ;\langle\cdot, \cdot\rangle)$ be a real inner product space generating the norm $\|\cdot\|$. The complexification $H_{\mathbb{C}}$ of $H$ is defined as a complex linear space $H \times H$ of all ordered pairs $(x, y)$, $x, y \in H$ endowed with the operations:

$$
\begin{aligned}
& (x, y)+\left(x^{\prime}, y^{\prime}\right):=\left(x+x^{\prime}, y+y^{\prime}\right), \quad x, x^{\prime}, y, y^{\prime} \in H \\
& (\sigma+i \tau) \cdot(x, y):=(\sigma x-\tau y, \tau x+\sigma y), \quad x, y \in H \quad \text { and } \quad \sigma, \tau \in \mathbb{R} .
\end{aligned}
$$

On $H_{\mathbb{C}}:=H \times H$, endowed with the above operations, one can now canonically define the scalar product $\langle\cdot, \cdot\rangle_{\mathbb{C}}$ by:

$$
\left\langle z, z^{\prime}\right\rangle_{\mathbb{C}}:=\left\langle x, x^{\prime}\right\rangle+\left\langle y, y^{\prime}\right\rangle+i\left[\left\langle x^{\prime}, y\right\rangle-\left\langle x, y^{\prime}\right\rangle\right]
$$

where $z=(x, y), z^{\prime}=\left(x^{\prime}, y^{\prime}\right) \in H_{\mathbb{C}}$. Obviously,

$$
\|z\|_{\mathbb{C}}^{2}=\|x\|^{2}+\|y\|^{2}, \quad z=(x, y) \in H_{\mathbb{C}} .
$$

One can also define the conjugate of a vector $z=(x, y)$ by $\bar{z}:=(x,-y)$. It is easy to see that, the elements of $H_{\mathbb{C}}$, under defined operations, behave as formal "complex" combinations $x+i y$ with $x, y \in H$. Because of this, we may write $z=x+i y$ instead of $z=(x, y)$. Thus, $\bar{z}=x-i y$. Under this setting, S. Kurepa [6] proved the following refinement of Schwarz's inequality:

$$
\left|\langle a, z\rangle_{\mathbb{C}}\right|^{2} \leq \frac{1}{2}\|a\|^{2}\left[\|z\|_{\mathbb{C}}^{2}+\left|\langle z, \bar{z}\rangle_{\mathbb{C}}\right|\right] \leq\|a\|^{2}\|z\|_{\mathbb{C}}^{2},
$$

for any $a \in H$ and $z \in H_{\mathbb{C}}$.

This was motivated by generalising the de Bruijn result for sequences of real and complex numbers obtained in [2].

The following result holds.

Theorem 2. Let $\left\{e_{j}\right\}_{j \in I}$ be a finite orthonormal family in the real inner product space $(H ;\langle\cdot, \cdot\rangle)$. Then for any $w \in H_{\mathbb{C}}$, where $\left(H_{\mathbb{C}} ;\langle\cdot, \cdot\rangle_{\mathbb{C}}\right)$ is the complexification of $(H ;\langle\cdot, \cdot\rangle)$, one has the following Bessel's type inequality:

$$
\begin{aligned}
\left|\sum_{j \in I}\left\langle w, e_{j}\right\rangle_{\mathbb{C}}^{2}\right| & \leq\left|\sum_{j \in I}\left\langle w, e_{j}\right\rangle_{\mathbb{C}}^{2}-\frac{1}{2}\langle w, \bar{w}\rangle_{\mathbb{C}}\right|+\frac{1}{2}\left|\langle w, \bar{w}\rangle_{\mathbb{C}}\right| \\
& \leq \frac{1}{2}\left[\|w\|_{\mathbb{C}}^{2}+\left|\langle w, \bar{w}\rangle_{\mathbb{C}}\right|\right] \leq\|w\|_{\mathbb{C}}^{2} .
\end{aligned}
$$

Proof. Define $f_{j} \in H_{\mathbb{C}}, f_{j}:=\left(e_{j}, 0\right), j \in I$. For any $k, j \in I$ we have

$$
\left\langle f_{i}, f_{j}\right\rangle_{\mathbb{C}}=\left\langle\left(e_{k}, 0\right),\left(e_{j}, 0\right)\right\rangle_{\mathbb{C}}=\left\langle e_{k}, e_{j}\right\rangle=\delta_{k j},
$$

therefore $\left\{f_{j}\right\}_{j \in I}$ is an orthonormal family in $\left(H_{\mathbb{C}} ;\langle\cdot, \cdot\rangle_{\mathbb{C}}\right)$. 
If we apply Theorem 1 for $\left(H_{\mathbb{C}} ;\langle\cdot, \cdot\rangle_{\mathbb{C}}\right), a=w, b=\bar{w}$, we may write:

$$
\left|\sum_{j \in I}\left\langle w, e_{j}\right\rangle_{\mathbb{C}}\left\langle e_{j}, \bar{w}\right\rangle_{\mathbb{C}}-\frac{1}{2}\langle w, \bar{w}\rangle_{\mathbb{C}}\right| \leq \frac{1}{2}\|w\|_{\mathbb{C}}\|\bar{w}\|_{\mathbb{C}} .
$$

However, for $w:=(x, y) \in H_{\mathbb{C}}$, we have $\bar{w}=(x,-y)$ and

$$
\left\langle e_{j}, \bar{w}\right\rangle_{\mathbb{C}}=\left\langle\left(e_{j}, 0\right),(x,-y)\right\rangle_{\mathbb{C}}=\left\langle e_{j}, x\right\rangle-i\left\langle e_{j},-y\right\rangle=\left\langle e_{j}, x\right\rangle+i\left\langle e_{j}, y\right\rangle
$$

and

$$
\left\langle w, e_{j}\right\rangle_{\mathbb{C}}=\left\langle(x, y),\left(e_{j}, 0\right)\right\rangle_{\mathbb{C}}=\left\langle e_{j}, x\right\rangle-i\left\langle e_{j},-y\right\rangle=\left\langle x, e_{j}\right\rangle+i\left\langle e_{j}, y\right\rangle
$$

for any $j \in I$. Thus $\left\langle e_{j}, \bar{w}\right\rangle=\left\langle w, e_{j}\right\rangle$ for each $j \in I$ and since

$$
\|w\|_{\mathbb{C}}=\|\bar{w}\|_{\mathbb{C}}=\left(\|x\|^{2}+\|y\|^{2}\right)^{\frac{1}{2}},
$$

we get from (3.4) that

$$
\left|\sum_{j \in I}\left\langle w, e_{j}\right\rangle_{\mathbb{C}}^{2}-\frac{1}{2}\langle w, \bar{w}\rangle_{\mathbb{C}}\right| \leq \frac{1}{2}\|w\|_{\mathbb{C}}^{2}
$$

Now, observe that the first inequality in (3.3) follows by the triangle inequality, the second is an obvious consequence of (3.5) and the last one is derived from Schwarz's result.

Remark 4. If the family $\left\{e_{j}\right\}_{j \in I}$ contains only a single element $e=\frac{x}{\|x\|}, x \in H$, $x \neq 0$, then from (3.3) we deduce (1.7), which, in its turn, provides a refinement of Kurepa's inequality (3.2).

\section{An Application for $L_{2}[-\pi, \pi]$}

It is well known that in the Hilbert space $L_{2}[-\pi, \pi]$ of all functions $f:[-\pi, \pi] \rightarrow \mathbb{C}$ with the property that $f$ is Lebesgue measurable on $[-\pi, \pi]$ and $\int_{-\pi}^{\pi}|f(t)|^{2} d t<\infty$, the set of functions

$$
\left\{\frac{1}{\sqrt{2 \pi}}, \frac{1}{\sqrt{\pi}} \cos t, \frac{1}{\sqrt{\pi}} \sin t, \ldots, \frac{1}{\sqrt{\pi}} \cos n t, \frac{1}{\sqrt{\pi}} \sin n t, \ldots\right\}
$$

is orthonormal.

If by trig $t$, we denote either $\sin t$ or $\cos t, t \in[-\pi, \pi]$, then on using the results from Sections 2 and 3, we may state the following inequality:

$$
\begin{aligned}
\mid \frac{1}{\pi} \sum_{k=1}^{n} \int_{-\pi}^{\pi} f(t) \operatorname{trig}(k t) d t \cdot \int_{-\pi}^{\pi} \overline{g(t)} \operatorname{trig}(k t) d t & -\left.\frac{1}{2} \int_{-\pi}^{\pi} f(t) \overline{g(t)} d t\right|^{2} \\
& \leq \frac{1}{4} \int_{-\pi}^{\pi}|f(t)|^{2} d t \int_{-\pi}^{\pi}|g(t)|^{2} d t,
\end{aligned}
$$


where all trig $(k t)$ is either $\sin k t$ or $\cos k t, k \in\{1, \ldots, n\}$ and $f \in L_{2}[-\pi, \pi]$.

This follows by Theorem 1 .

If one uses Corollary 1, then one can state the following chain of inequalities

$$
\begin{aligned}
& \left|\frac{1}{\pi} \sum_{k=1}^{n} \int_{-\pi}^{\pi} f(t) \operatorname{trig}(k t) d t \cdot \int_{-\pi}^{\pi} \overline{g(t)} \operatorname{trig}(k t) d t\right| \\
& \leq\left|\frac{1}{\pi} \sum_{k=1}^{n} \int_{-\pi}^{\pi} f(t) \operatorname{trig}(k t) d t \cdot \int_{-\pi}^{\pi} \overline{g(t)} \operatorname{trig}(k t) d t-\frac{1}{2} \int_{-\pi}^{\pi} f(t) \overline{g(t)} d t\right| \\
& \quad \quad+\frac{1}{2}\left|\int_{-\pi}^{\pi} f(t) \overline{g(t)} d t\right| \\
& \leq \frac{1}{2}\left[\left(\int_{-\pi}^{\pi}|f(t)|^{2} d t \int_{-\pi}^{\pi}|g(t)|^{2} d t\right)^{\frac{1}{2}}+\left|\int_{-\pi}^{\pi} f(t) \overline{g(t)} d t\right|\right] \\
& \leq\left(\int_{-\pi}^{\pi}|f(t)|^{2} d t \int_{-\pi}^{\pi}|g(t)|^{2} d t\right)^{\frac{1}{2}},
\end{aligned}
$$

where $f \in L_{2}[-\pi, \pi]$.

Finally, by employing Theorem 2, we may state:

$$
\begin{aligned}
& \frac{1}{\pi}\left|\sum_{k=1}^{n}\left[\int_{-\pi}^{\pi} f(t) \operatorname{trig}(k t) d t\right]^{2}\right| \\
& \leq\left|\frac{1}{\pi} \sum_{k=1}^{n}\left[\int_{-\pi}^{\pi} f(t) \operatorname{trig}(k t) d t\right]^{2}-\frac{1}{2} \int_{-\pi}^{\pi} f^{2}(t) d t\right|+\frac{1}{2}\left|\int_{-\pi}^{\pi} f^{2}(t) d t\right| \\
& \leq \frac{1}{2}\left[\int_{-\pi}^{\pi}|f(t)|^{2} d t+\left|\int_{-\pi}^{\pi} f^{2}(t) d t\right|\right] \leq \int_{-\pi}^{\pi}|f(t)|^{2} d t,
\end{aligned}
$$

where $f \in L_{2}[-\pi, \pi]$.

\section{References}

[1] C. Blatter, Zur Riemannschen Geometrie im Grossen auf dem Möbiusband (German), Compositio Math. 15 (1961) 88-107.

[2] N. G. de Bruijn, Problem 12, Wisk. Opgaven 21(1960), 12-14.

[3] M. L. Buzano, Generalizzazione della disiguaglianza di Cauchy-Schwarz (Italian), Rend. Sem. Mat. Univ. e Politech. Torino 31 (1971/73), 405-409 (1974).

[4] S. S. Dragomir, Refinements of Buzano's and Kurepa's inequalities in inner product spaces, Facta Univ. Ser. Math. Inf. 20(2005), 65-73.

[5] M. Fujii and F. Kubo, Buzano's inequality and bounds for roots of algebraic equations, Proc. Amer. Math. Soc. 117(1993), 359-361.

[6] S. Kurepa, On the Buniakowsky-Cauchy-Schwarz inequality, Glasnick Mathematički 1(21) (2)(1966), 147-158. 
[7] J. E. Pečarić, On some classical inequalities in unitary spaces, Mat. Bilten, (Macedonia) 16 (1992), 63-72.

[8] T. Precupanu, On a generalisation of Cauchy-Buniakowski-Schwarz inequality, Anal. St. Univ. "Al. I. Cuza" Iaşi, 22(1976), 173-175.

[9] U. Richard, Sur des inégalités du type Wirtinger et leurs application aux équationes différentielles ordinaires, Colloquium of Analysis held in Rio de Janeiro, August, 1972, pp.233-244.

School of Computer Science and Mathematics, Victoria University, PO Box 14428, MCMC 8001, VIC, Australia.

E-mail: sever@csm.vu.edu.au 